\title{
Association of sarcopenic obesity with the risk of all-cause mortality among adults over a broad range of different settings: a updated meta-analysis
}

Xiaoming Zhang ${ }^{1 \dagger}$, Xiaohua Xie ${ }^{2 \dagger}$, Qingli Dou', Chenyun Liu', Wenwu Zhang ${ }^{1}$, Yunzhi Yang ${ }^{3}$, Renli Deng ${ }^{4^{*}}$ (D) and Andy S. K. Cheng ${ }^{5}$

\begin{abstract}
Background: Previous cohort studies investigating the association between sarcopenic obesity (SO) and all-cause mortality among adult people have been inconsistent. We performed a meta-analysis to determine if SO is a predictor of all-cause mortality.

Methods: Prospective cohort studies that evaluated the association between SO and mortality in older people were identified via a systematic search of three electronic databases (PubMed, EMBASE, and the Cochrane Library). A random-effects model was applied to combine the results. We considered the methods recommeded by consensuses (dual X-ray absorptiometry,bio-impedancemetry, anthropometric measures or CT scan) to assess sarcopenic obesity.

Results: Of the 603 studies identified through the systematic review, 23 (Participants: 50866) were included in the meta-analysis. The mean age ranged from 50 to 82.5 years.SO was significantly associated with a higher risk of allcause mortality among adult people (pooled $\mathrm{HR}=1.21,95 \%$ confidence interval $[95 \% \mathrm{Cl}]=1.10-1.32, p<0.001, P^{2}=$ 64.3\%). Furthermore, the subgroup analysis of participants showed that SO was associated with all-cause mortality (pooled HR $=1.14,95 \% \mathrm{Cl}: 1.06-1.23$ ) among community-dwelling adult people; similarly, this association was found in hospitalized patients (pooled $\mathrm{HR}=1.65,95 \% \mathrm{Cl}: 1.17-2.33$ ). Moreover, the subgroup analysis demonstrated that SO was associated with all-cause mortality when using skeletal muscle mass (SMM) criteria, muscle strength criteria, and skeletal muscle index (SMI) criteria ( $\mathrm{HR}=1.12,95 \% \mathrm{Cl}: 1.01-1.23 ; \mathrm{HR}=1.18,95 \% \mathrm{Cl}: 1.05-1.33$; and $\mathrm{HR}=1.53$, 95\% Cl: 1.13-2.07, respectively). In addition, we analyzed SO on the basis of obesity definition and demonstrated that participants with a SO diagnosis based on waist circumference (WC) $(H R=1.24,95 \%$ Cl: 1.09-1.40), body mass index (BMI) $(\mathrm{HR}=1.29,95 \% \mathrm{Cl}: 1.04-1.59)$, or visceral fat area $(\mathrm{HR}=2.54,95 \% \mathrm{Cl}: 1.83-3.53)$ have a significantly increase mortality risk compared with those without SO.
\end{abstract}

Conclusion: Based on our update of existing scientific researches, SO is a significant predictor of all-cause mortality among older people, particularly hospitalized patients. Therefore, it is important to diagnose SO and to treat the condition to reduce mortality rates among older people.

Keywords: Sarcopenic obesity, All-cause mortality, Older adults, Meta-analysis

\footnotetext{
* Correspondence: zhangmuxi0310@163.com

${ }^{+}$Xiaoming Zhang and Xiaohua Xie are co-first authors

${ }^{4}$ Department of Nursing, The Fifth Affiliated Hospital of Zunyi Medical

University, Zhuhai, China

Full list of author information is available at the end of the article
}

(C) The Author(s). 2019 Open Access This article is distributed under the terms of the Creative Commons Attribution 4.0 International License (http://creativecommons.org/licenses/by/4.0/), which permits unrestricted use, distribution, and reproduction in any medium, provided you give appropriate credit to the original author(s) and the source, provide a link to the Creative Commons license, and indicate if changes were made. The Creative Commons Public Domain Dedication waiver (http://creativecommons.org/publicdomain/zero/1.0/) applies to the data made available in this article, unless otherwise stated. 


\section{Background}

Sarcopenia is defined as a condition of age-related loss of muscle mass and muscle strength with functional impairment in terms of physical performance, and it has been associated with a series of adverse health consequences among older adults [1], including falls [1] and fractures [2], decreased mobility [3], depression [4], poor quality of life [5], hospitalization [6], and mortality [7]. The prevalence of obesity in the adult people of the world is rising alarmingly [8], potentially augmenting supplemental conditions and increasing the risk of adverse health outcomes. According to some studies, obesity increases the chance of multiple chronic health conditions and is also related to increased risk of death $[9,10]$. Studies found that sarcopenia is often accompanied by an increase in adipose tissue, and this condition was defined as sarcopenic obesity (SO) [11]. In addition, research found that sarcopenia and obesity may have common inflammatory pathways [12]. Given the fact that both sarcopenia and obesity would increase the risk of all-cause mortality $[13,14]$, it is hypothesized that the coexistence of sarcopenia and obesity may synergistically aggravate the risk of mortality.

Recently, multiple studies have found that SO is a predictor of all-cause mortality among community-dwelling older people $[15,16]$. However, some other studies have found no significant association between $\mathrm{SO}$ and all-cause mortality $[17,18]$. In a recent meta-analysis study, Tian et al. [19] analyzed SO and all-cause mortality and concluded that older people with SO, particularly males, are associated with a $24 \%$ increase in the risk of all-cause mortality compared with those without SO. However, the authors did not perform a subgroup analysis of the types of participant. Therefore, it is unclear whether SO increases the risk of all-cause mortality among community-dwelling adults. In terms of the function of community-dwelling people, it would lead to the sub-group analysis. Furthermore, more prospective studies about this issue have been published since 2015 given that this is a rapidly progressing research field $[15,18,20]$.

Given the observed contradictory relationship between SO and all-cause mortality among community-dwelling adults in some studies, further studies are needed. Therefore, this updated meta-analysis aimed to identify and compare prospective cohort studies examining the association between SO and all-cause mortality among adults according to the meta-analysis Of Observational Studies in Epidemiology (MOOSE) guidelines.

\section{Materials and methods}

This systematic review was conducted and reported according to the MOOSE guidelines [21].

\section{Search strategy and selection criteria}

We performed a systematic literature search in MEDLINE (via PubMed 1946 to October 2018), EMBASE (via EMBASE October 2018), and Cochrane CENTRAL Library (via Cochrane Library October 2018) and screened the relevant study that reported the association between SO and all-cause mortality. The search strategy included a combination of keywords and $\mathrm{MeSH}$ terms, such as mortality (mortality $\left.{ }^{*}\right)$, OR death (death*), OR survival (survival*) and sarcopenia (sarcopenia*) and obesity (obesity*).In addition, other search strategy of subject terms and truncation symbols were also used in order to find all related articles. We searched the potential gray studies through Google Scholar database and the search strategy was showed in Additional file 1.

\section{Study selection}

Two investigators (XMZ and CYL) independently reviewed the studies by screening each title and abstract and then confirmed the including study by full text. If there was a disagreement regarding the inclusion or exclusion of a study, these issues were discussed with the third investigator until a consensus was achieved.

\section{Inclusion and exclusion criteria}

The following eligibility and exclusion criteria were prespecified. Studies had to meet the following three inclusion criteria: (1) prospective cohort studies; (2) studies investigating the association between $\mathrm{SO}$ and mortality; and (3) the primary or secondary outcome of interest was all-cause mortality; The exclusion criteria were as follows: (1) irrelevant type of articles: conference abstract, and letters and review articles; (3) insufficient data; (4) studies written in languages other than English; and (5) no clear definition of sarcopenia.

\section{Data extraction}

The data from the selected studies using a standardized data-abstraction form was independently abstracted by two investigators (QLD and RLD). The following information that consisted of author, country, year of publication, demographic characteristics of participants (e.g, age, sample size, proportion of males), measurement methods of sarcopenia, and follow-up period were extracted from the included papers. The principle was that two reviewers cross-checked all the extracted data. Disagreements were resolved by discussion until a consensus was achieved. Those studies included different definitions of SO to show HR or displayed the HR by gender would extract by each definition as eligible studies.

\section{Assessment of risk of bias}

Two reviewers (YY, WWZ) independently assessed the risk of bias of including studies by according to the Newcastle-Ottawa Scale (NOS) that which includes six aspects: (1) representativeness of the exposed cohort, (2) comparability of group, (3) blinding of investigators who 
measured outcomes, (4) duration and completeness of follow-up, (5) contamination bias, and (6) other potential sources of bias [22]. The maximum total score of the scale is 9 points. We regarded a study whose total score was more than or equal to 5 points as a high quality research.

\section{Statistical analysis}

Two authors (XMZ, QLD) independently use STATA version 14.0 (Stata Corp, College Station, TX, USA) to analyze all the data. Hazard ratios (HRs), and their 95\% CIs of mortality for SO compared with non-sarcopenic-obesity, were extracted from studies for future meta-analysis. We considered the adjusted HR for potential confounder models as the final result in our meta-analysis in order to reduce confounding effects. When there were more than two studies in the subgroup, we conducted a subgroup analysis of gender, setting, and different SO definitions. We used the Cochran's Q statistic using chi-square and $I^{2}$ statistics to examine the heterogeneity among the included studies and $I^{2}$ values of 25, 50, and 75\% was regarded as low, moderate, and high heterogeneity, respectively. By analyzing all methods of included studies, it was acknowledged that there was a heterogeneity in our study due to many different aspects,for instance,various criteria used to evaluate SO, different types of participant, and different lengths of follow-up. Therefore, a random-effects model was applied, regardless of the heterogeneity, to obtain more conservative but reliability results. Results were showed using forest plots, and the Begg's test was conducted to assess the potential publication bias. We also performed sensitivity analyses that assessed whether the overall estimate effect size was stability.

\section{Results}

\section{Search results}

Our literature search strategy initially identified 603 articles. After the removal of duplicate files, 497 articles were screened to determine whether they were eligible. We screened the titles and abstracts of these articles and removed non-related articles and finally 36 publications remained for further screening. Of these articles, 13 were deleted because they were non-cohort studies (e.g., review articles, conference abstract, cross-sectional study, letter), and six were removed because they had an irrelevant subject (dynapenic abdominal obesity, cardiovascular disease) as their outcome. A total of 17 articles with 23 eligible studies were finalized on the basis of the predefined inclusion and exclusion criteria for meta-analysis. Figure 1 shows the details of our literature search and selection process.

\section{Included studies}

Twenty-three prospective cohort studies [15-18, 22-34], with the total of 50,866 participants, were included in our meta-analysis. We summarized the detailed description of the characteristics of these 23 prospective cohort studies (from 17 articles) in Table 1. Of these 23 studies, five were conducted in the USA [16, 26], one in Portugal [33], one in Australia [23], four in Japan [15, 27, 28, 30], two in the UK [18, 24], one in Finland [22], and two in China [29]. All the studies regarded all-cause mortality as a clinical outcome. Three studies used different SO definitions, and three articles reported gender HR and data were used for HR extraction for men and women, respectively. There are several diagnosis criteria of SO in our meta-analysis. One study used the midarm muscle circumference (MAMC)-based definition of SO [24], eight used the SMM-based definition [15, 24, 25, 27, 28], three used the muscle strength (MS)-based definition [17, 18, 22], three used the appendicular lean mass (ALM)/BMI-based definition $[16,23]$, and eight used the SMI-based definition [26, 29-34]. Meanwhile, four studies used body mass index (BMI) to define obesity $[18,22,32,34]$, three used waist circumference (WC) $[15,17,24]$, nine used body composition body fat (BF) $[15,16,25,26,31]$, and five used visceral fat area [27-30, 33]. In addition, all of the included studies adjusted for diverse confounding factors. Followup periods ranged from 3 years to 33 years.

\section{Quality assessment}

A detailed description of the methodological quality assessment using NOS was provided in Table 2. Scores ranged from 7 to 8 , and nine studies scored more than 7 points.

\section{Sarcopenic obesity as a predictor of all-cause mortality Meta-analysis of studies}

Twenty-three prospective cohort studies (from 17 articles) examined the relationship between $\mathrm{SO}$ and mortality in adult people. A random-effects model was applied to calculate the pooled HR values. As shown in Fig. 2, the pooled HRs of all-cause mortality for $\mathrm{SO}$ versus non-sarcopenic non-obese was $1.21(95 \% \mathrm{CI}=1.10-1.32$, $p<0.001)$, and significant heterogeneity was found across these studies (Q-value $=48.75$, degree of freedom $=16, \mathrm{I}^{2}=64.3 \%, \mathrm{p}<0.001$ ).

\section{Subgroup analysis}

The 23 studies with a HR of all-cause mortality risk for people were further analyzed by subgroup due to medium heterogeneity. Figure 3 shows the pooled effect by study setting, which showed that among community-dwelling adults with $\mathrm{SO}$ had a significantly increased risk of mortality (HR $=1.14,95 \%$ CI: 1.06-1.23) compared to those with non-sarcopenic obesity; a similar result was found for hospitalized patients ( $\mathrm{HR}=1.65,95 \% \mathrm{CI}: 1.17-2.33)$. Figure 4 shows the pooled effect by five definitions of sarcopenic obesity. Overall, using the MS-based definition, the participant with SO had a higher risk of mortality, compared with those without SO $(\mathrm{HR}=1.18,95 \%$ CI: 1.05-1.33). 


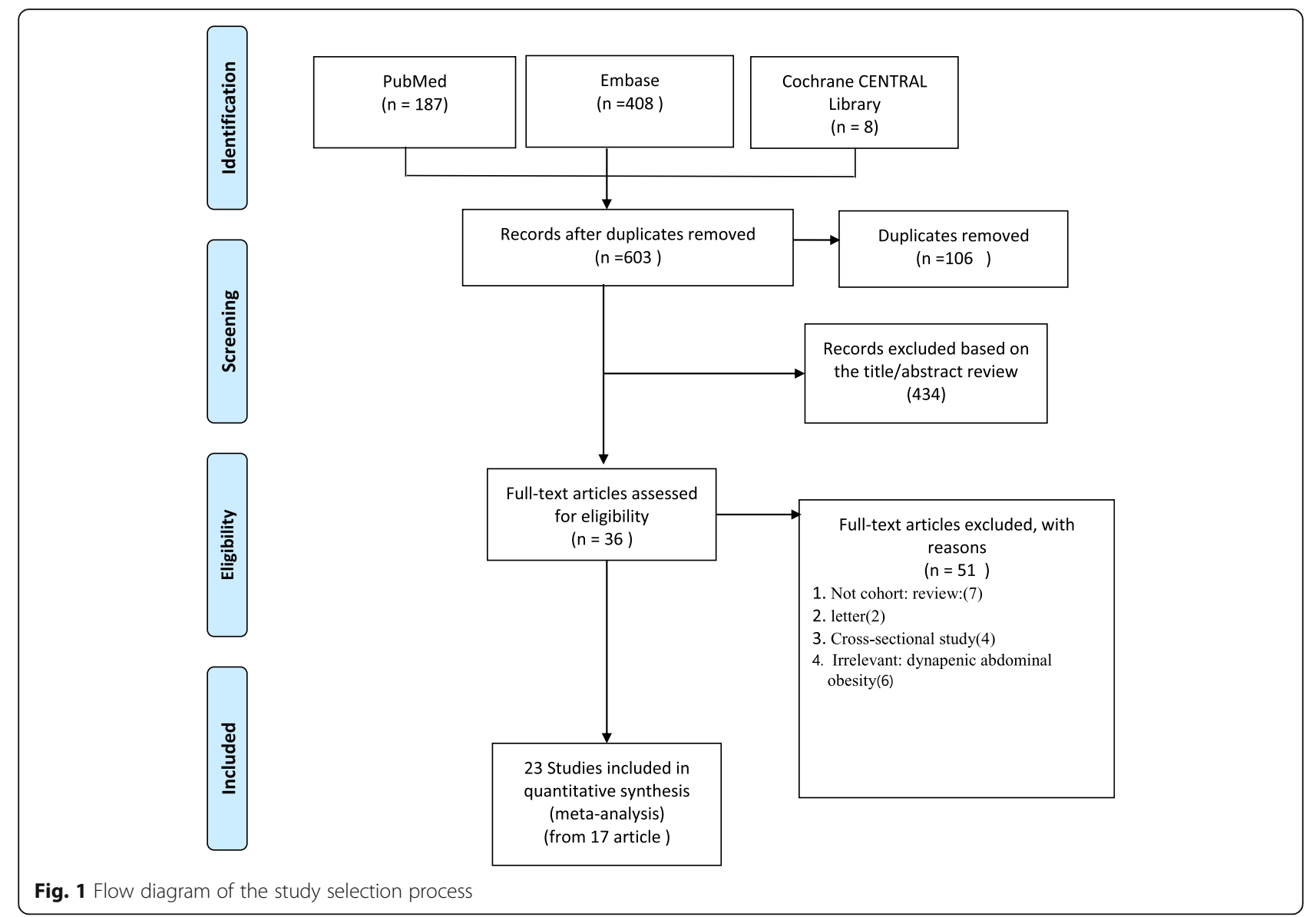

Similarly, as for SMM-based definition and SMI-based definition, the SO was associated with higher risk of death $(\mathrm{HR}=1.12$, 95\% CI: $1.01-1.23$; HR $=1.53$, 95\% CI: $1.13-$ 2.07 , respectively). However, in the three studies using the ALM/BMI definition, SO was not statistically associated with an increased risk of mortality ( $\mathrm{HR}=1.05,95 \% \mathrm{CI}$ : 0.84-1.32). In addition, participants with WC-based SO, BMI-based SO, visceral fat area based SO, or BF-based SO had a significantly increased risk of mortality compared with those without $\mathrm{SO}(\mathrm{HR}=1.24,95 \% \mathrm{CI}: 1.09-1.40$; $\mathrm{HR}=1.29$, 95\% CI: $1.04-1.59$; $\mathrm{HR}=2.54,95 \%$ CI: $1.83-$ 3.53; $\mathrm{HR}=1.12,95 \% \mathrm{CI}: 1.01-1.24$, respectively). However, an increased risk of mortality was not found in fat-massbased SO (HR = 0.94, 95\% CI: 0.80-1.09) (Fig. 5). Subgroup analyses of gender (Fig. 6) and duration of follow-up (Fig. 7) were performed. The results showed that the corresponding risk estimates were 1.14 (95\% CI: $1.05-1.25)$ and 1.29 (95\% CI: 1.09-1.54) for a follow-up duration of $\geq 10$ years and $<10$ years, respectively.

\section{Publication bias assessment}

The results of the Begger's tests $(p=0.02)$ suggested that there may be some publication bias in our study (Additional file 2: Figure S1). However, when we applied the trim-and-fill analyses to assess publication bias, the results showed that both the trimmed studies and the filled studies were similar, which indicates that the pooled HR was relatively stable (Additional file 3: Figure S2).

\section{Sensitivity analyses}

We performed sensitivity analyses of $\mathrm{SO}$ and mortality to evaluate the stability of pooled results. The results of the sensitivity analyses confirmed that there were no statistically significant changes (Additional file 4: Figure S3). In addition, We found the sensitivity analysis of age group showed that Participants age $50-70$ years with SO was associated with all-cause mortality (pooled $\mathrm{HR}=1.32,95 \%$ CI: 1.14-1.53); similarly, participants with SO aged 70 years and older did have a marginally association (pooled HR = 1.10, 95\% CI: 1.00-1.21) (Additional file 5: Figure S4).

\section{Discussion}

Our study found that people with SO significantly increase the risk of mortality with a 1.21-fold risk comparing to non-sarcopenic non-obese. The included studies were implemented in various countries and had a range of follow-up durations and five different SO definitions. The pooled HR was consistent with the sensitivity 


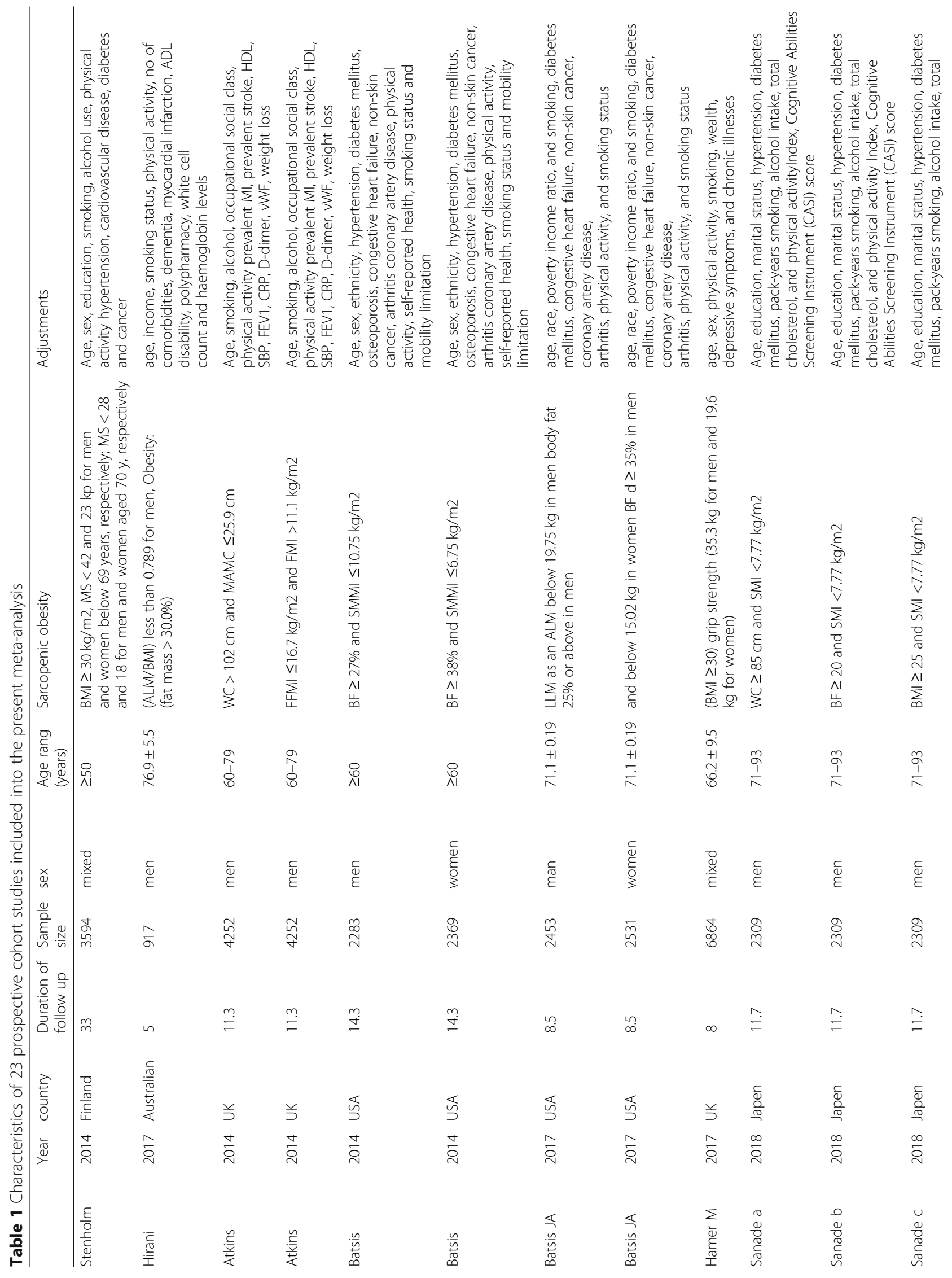




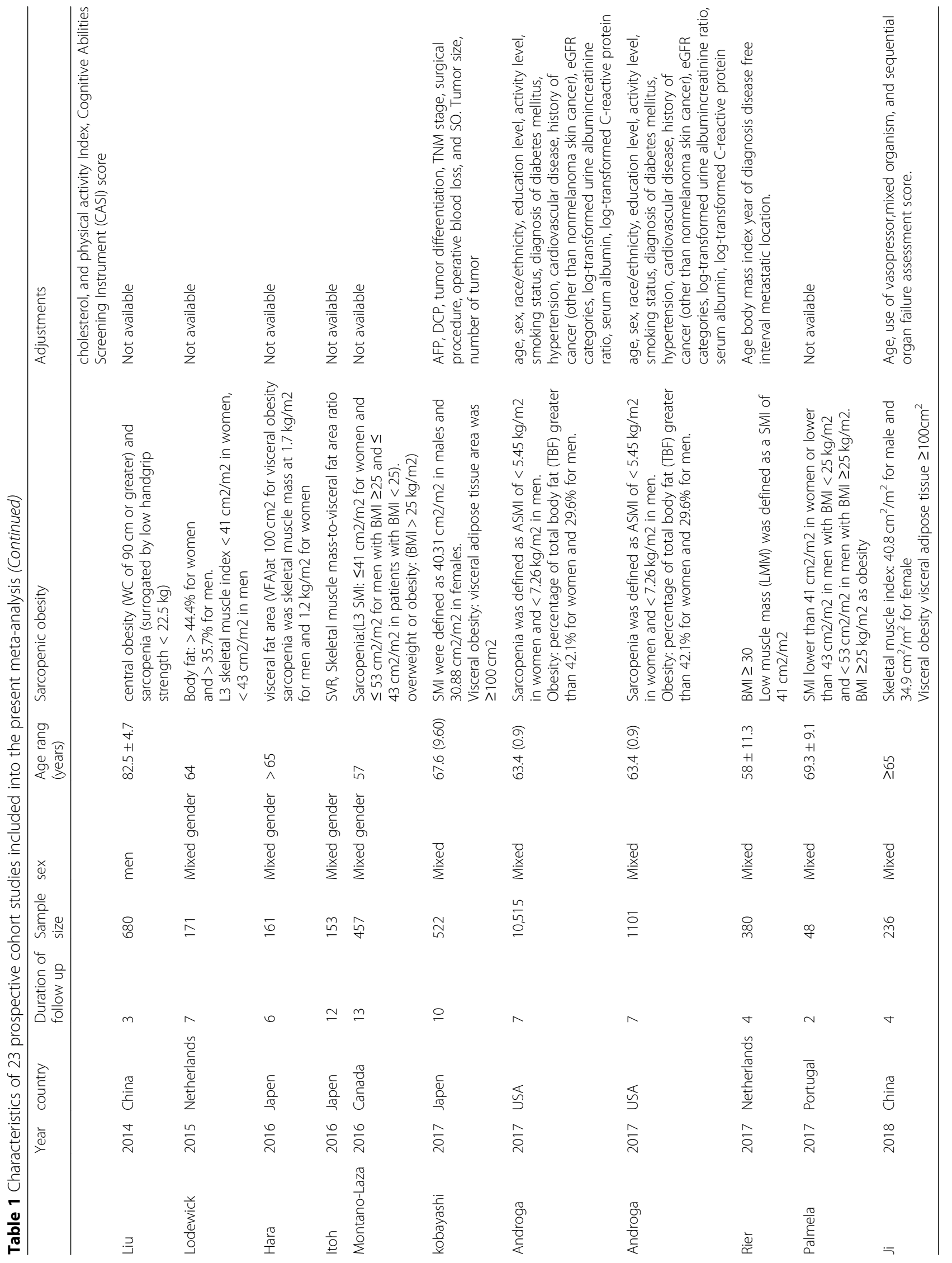


Table 2 Quality (Newcastle-Ottawa Scale) of the studies included in the meta-analysis

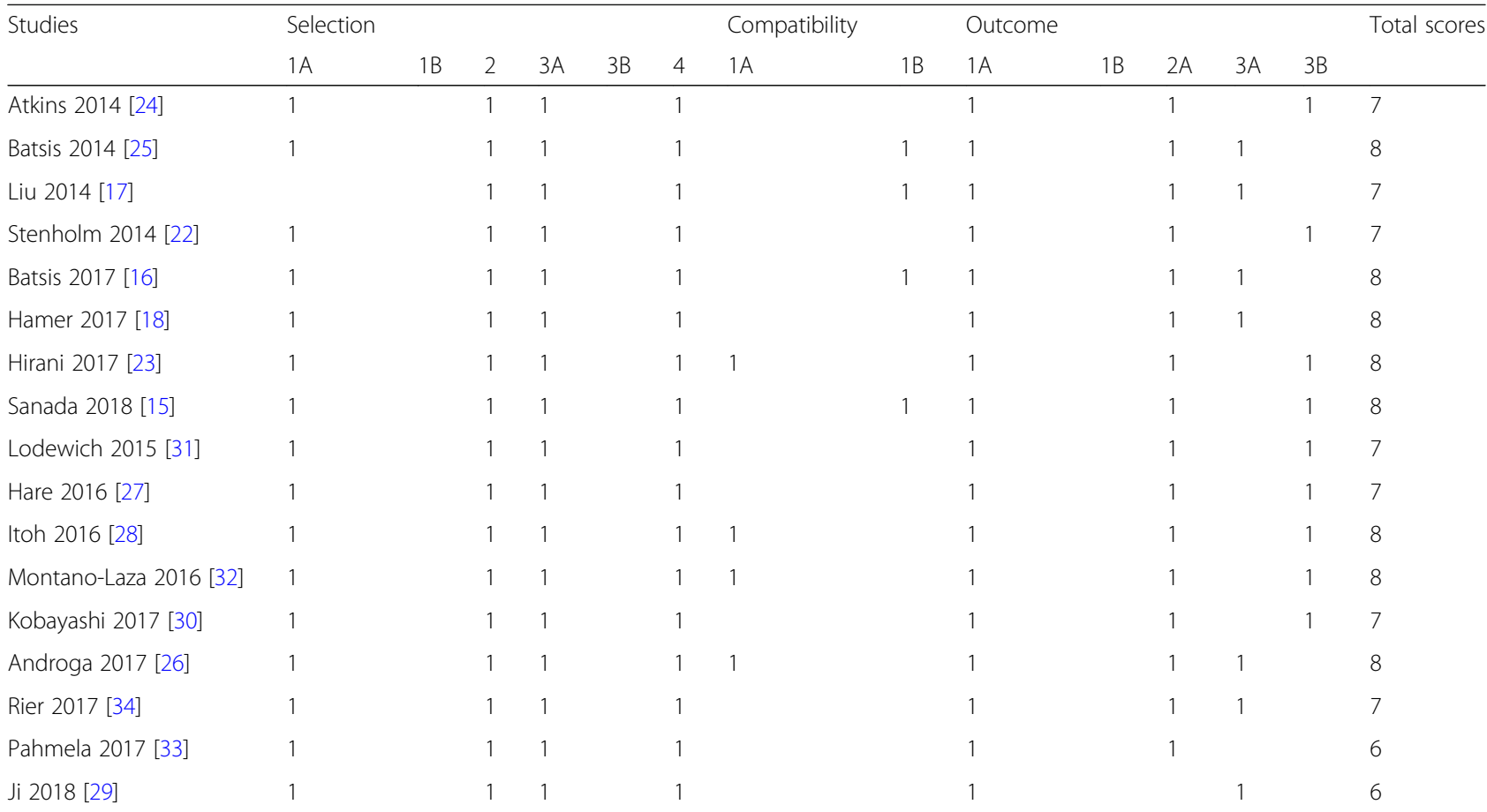

Study

ID

Atkins 2014a

Atkins 2014b

Batsis 2014a

Batsis 2014b

Liu 2014

Stenholm 2014

Lodewick 2015

Hara 2016

Itoh 2016

Montano 2016

Batsis 2017a

Batsis 2017b

Hirani 2017

Hamer 2017

kobayashi 2017

Androga 2017a

Androga 2017b

Palmela 2017

Rier 2017

Sanada 2017a

Sanada 2017b

Sanada 2017c

Ji 2018

Overall (I-squared $=64.3 \%, p=0.000$ )

NOTE: Weights are from random effects analysis
$\%$

HR $(95 \%$ CI) Weight

$1.44(1.10,1.89) \quad 4.84$

$0.98(0.80,1.20) \quad 5.93$

$1.25(0.99,1.58) \quad 5.42$

$0.98(0.79,1.22) \quad 5.68$

$1.19(0.84,1.67) \quad 3.86$

$1.17(1.01,1.35) \quad 6.89$

$0.87(0.51,1.25) \quad 2.80$

$2.14(0.65,7.01) \quad 0.56$

$2.58(1.17,5.52) \quad 1.21$

$1.72(1.30,2.28) \quad 4.69$

$0.99(0.85,1.16) \quad 6.72$

$1.31(1.11,1.55) \quad 6.53$

$0.88(0.70,1.11) \quad 5.47$

$1.22(0.93,1.61) \quad 4.79$

$2.03(1.23,3.22) \quad 2.55$

$1.57(1.19,2.05) \quad 4.82$

$0.97(0.70,1.35) \quad 4.05$

$3.67(1.68,8.05) \quad 1.19$

$0.87(0.40,1.88) \quad 1.21$

$1.19(1.02,1.38) \quad 6.79$

$1.03(0.86,1.23) \quad 6.33$

$1.15(1.00,1.33) \quad 6.93$

$4.20(1.50,11.60) \quad 0.74$

$1.21(1.10,1.32) \quad 100.00$

Fig. 2 Forest plots for the risk of all-cause mortality among adults with sarcopenic obesity 


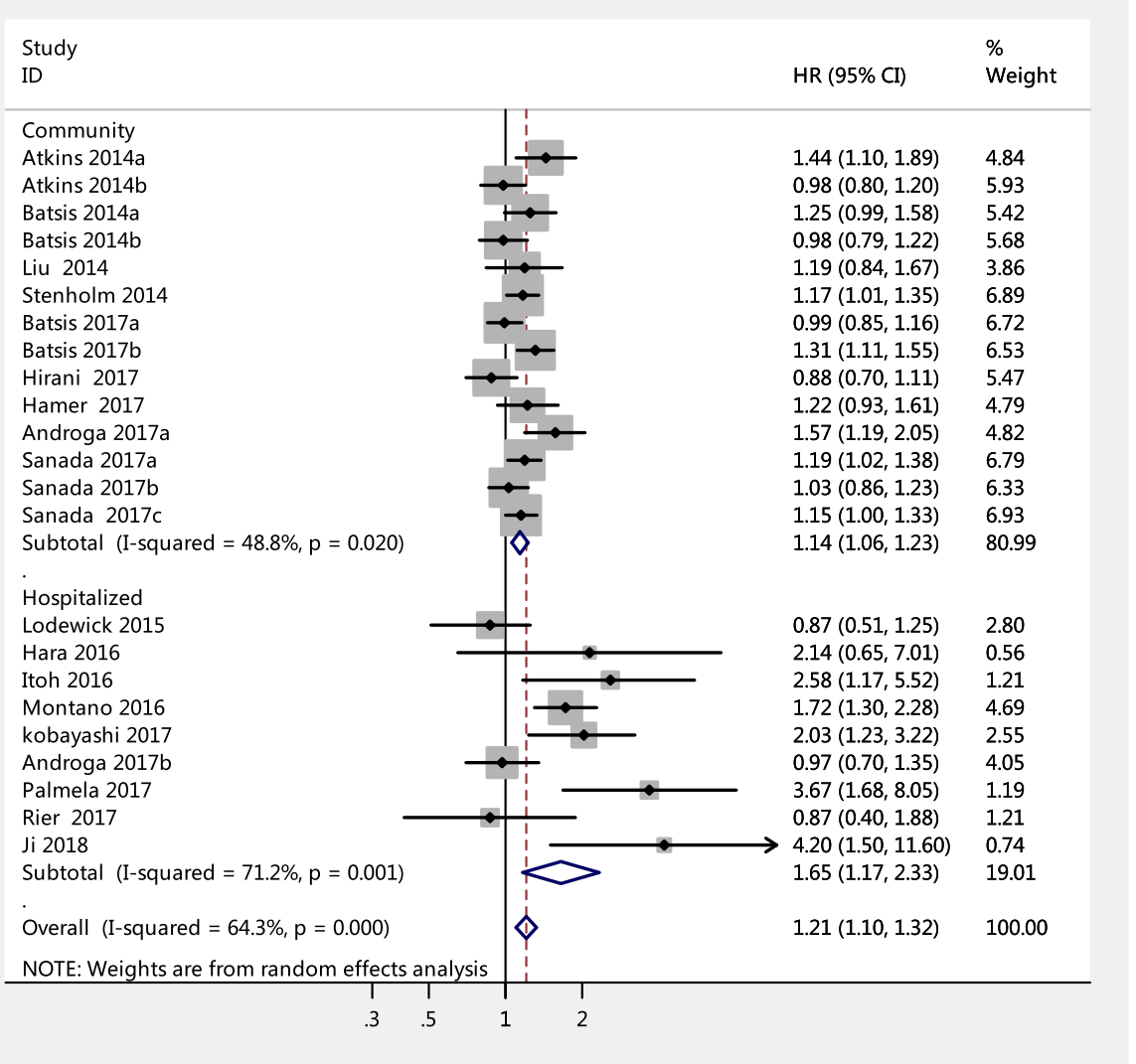

Fig. 3 Subgroup analysis of setting for the risk of all-cause mortality among adults with sarcopenic obesity

analyses. However, the results of the Begger's tests indicated that there may have been publication bias $(P=$ $0.02)$. In order to identify this influence, we performed trim-and-fill analyses, and the results showed similar results after incorporating the hypothesized studies to achieve the symmetry of the funnel plots, which showed that our pooled results were stable and reliable. In addition, the results of the subgroup analyses all showed that $\mathrm{SO}$ significantly increased the risk of mortality, except in the SO groups using the ALM/BMI and fat mass definitions of SO. Our findings emphasize that $\mathrm{SO}$ is an significant risk of mortality in the adult people, especially for hospitalized patients, and that preventative strategies aiming to SO are urgently needed to reduce the rate of mortality among adult people.

Tian [19] and colleagues performed a systematic review and meta-analysis of the association of $\mathrm{SO}$ with mortality in 2016. This paper was comprehensive and the method was appropriate. However, this review did not perform subgroup analyses of the participants. There is a big difference between community-dwelling people and disease-specific populations [36]. People with SO in disease-specific populations [35] may have an increased risk of death compared to relatively healthy community- dwelling seniors, which may overestimate or underestimate the results. Therefore, it is important to identify the role of SO among community-dwelling people and hospitalized people. In addition, Tian and colleagues did not perform an assessment of risk bias using the Newcastle Ottawa Scale, which may make it hard to provide the necessary "lever of evidence." Therefore, its results may not be applicable to people in the community. The methodological quality of our review was good in that it included publication bias evaluation, heterogeneity testing, sensitivity analysis, and rigorous subgroup analysis, which may lead to more accurate conclusions.

In this study, we confirmed a significant association between $\mathrm{SO}$ and the risk of all-cause mortality among adult people; however, we found that the level of heterogeneity among the studies was medium $\left(I^{2}=64.3 \%\right)$. After the subgroup analysis of obesity definitions, we found that the heterogeneity in our study was perfect $\left(I^{2}=0 \%\right)$ when SO was defined by WC, fat mass, and visceral fat area. A similar phenomenon was found for SMM-based SO and muscle strength based SO. Even though heterogeneity was not reduced in ALM/BMIbased SO and BMI-based obesity, we believe this may be explained by the different cut-off values used in the 


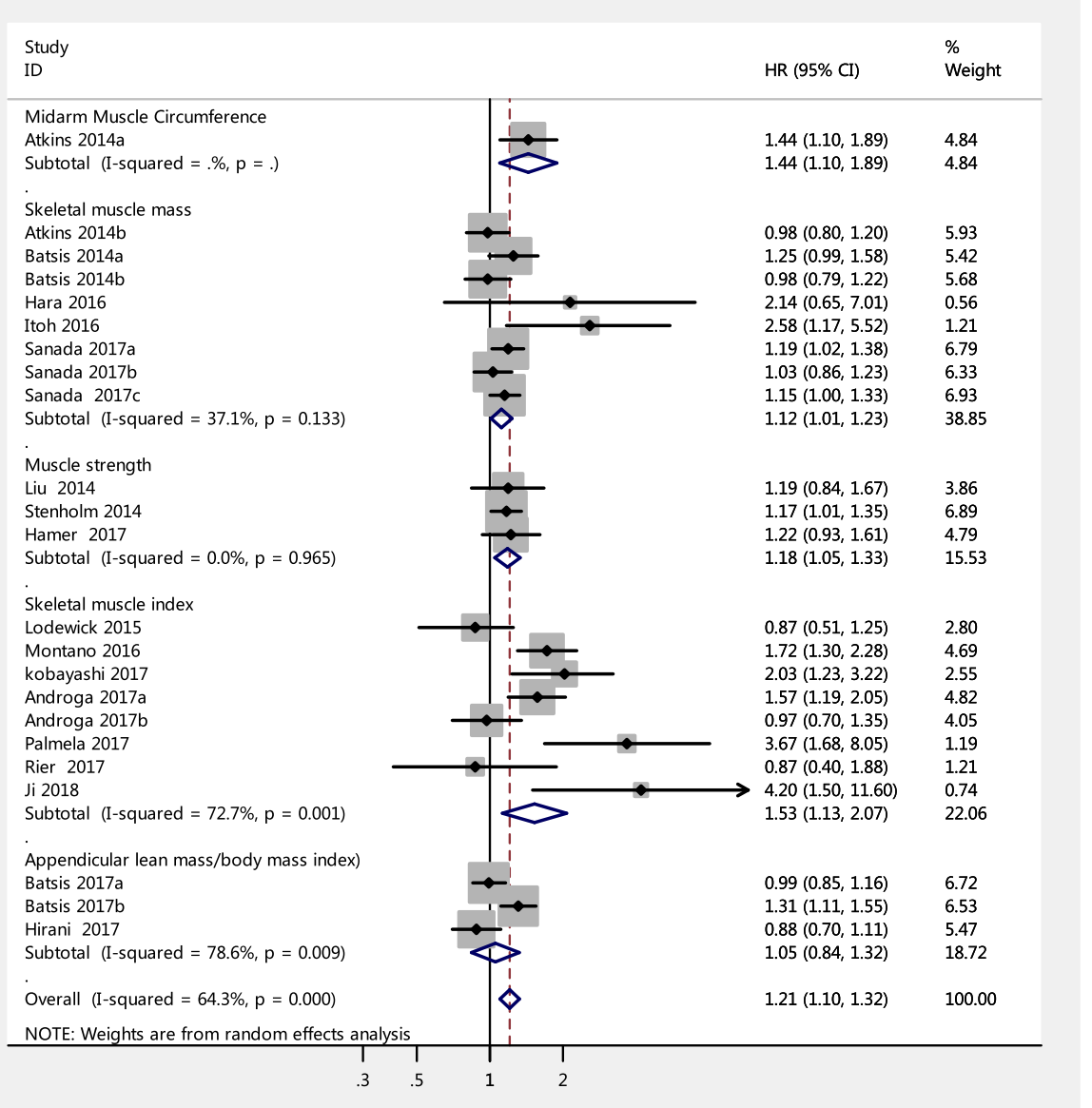

Fig. 4 Forest plots for the risk of all-cause mortality among adults associated with sarcopenic obesity according to different sarcopenia definitions

original studies. Therefore, it is reasonable to assume that the heterogeneity was mainly caused by the various definitions of SO and the different cut-off values.

The subgroup analysis of SO criteria showed that participants with SO had an increased the risk of mortality compared with normal people, and the association may not be significantly affected by the definition of SO, except when the ALM/BMI-based definition is used. This meta-analysis found that participants with $\mathrm{SO}$ defined by SMI had a significantly increased risk of mortality (53\%) compared with those without SO, which suggests that SMI-based SO can provide relevant diagnostic criteria for sarcopenia to assess the mortality of adult people. The reason for this result was our inclusion of studies that used CT-imaging to determine muscle mass and then calculating SMI. According to the consensus guidelines of the European Working Group on Sarcopenia in People, muscle mass measured by CT-imaging is the golden standard for measuring muscle parameters, especially among hospitalized patients. However, circumstances in the community are different. Because of scarce medical facilities, it is difficult for community seniors to have their muscle size checked by CT-imaging. Therefore, it is imperative to develop a portable alternative to CT-imaging. Our study suggests that the combined HR (pooled $\mathrm{HR}=1.44$, 95\% CI: 1.10-1.89) was relatively higher when using the MAMC-based definition of SO, which suggests that this definition of SO can provide relevant diagnosis criteria for sarcopenia to assess the mortality risk of community-dwelling adults; this result is in line with a previous study [19]. Several cohort studies [37-39] confirmed a significant association between low MAMC and increased risk of mortality. However, this association was not found for ALM/ BMI-based SO. The possible reason maybe the Foundation for the National Institutes of Health (FNIH) [40] definition of sarcopenia, which will indicate the prevalence of SO much less. In a cohort study in Switzerland including 913 participants after a 3-year follow-up, Melany Hars [41] found that the prevalence of sarcopenia was $11.2 \%$ using the European Working Group on Sarcopenia in Older People (EWGSOP) definition, 


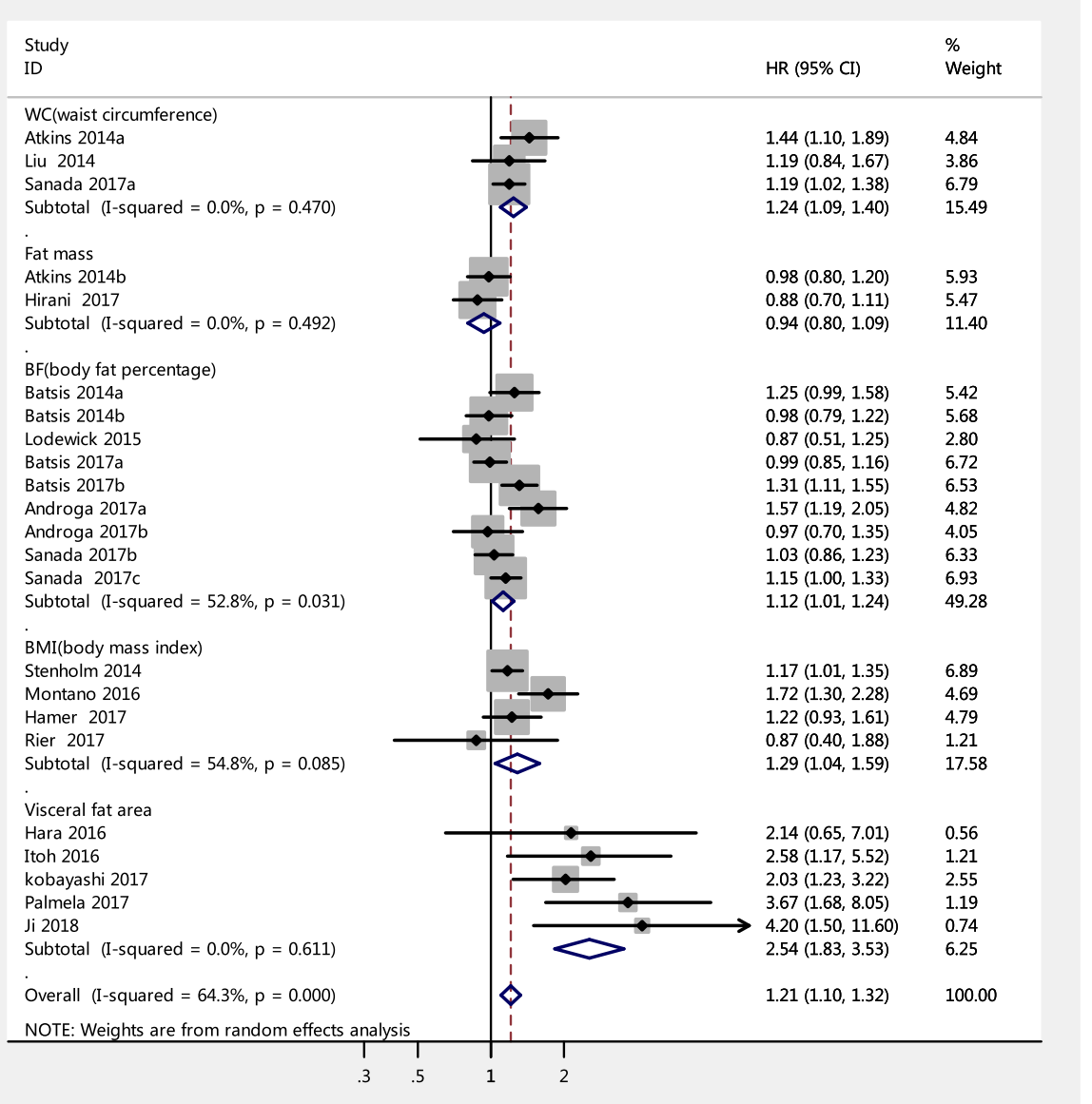

Fig. 5 Forest plots for the risk of all-cause mortality among adults associated with obesity according to different obesity definitions

whereas the prevalence of sarcopenia was $3.5 \%$ when the FNIH was used to define sarcopenia. Therefore, we believe that the prevalence of SO measured using the FNIH definition produces no significant association between SO and mortality. Future cohort studies are needed to investigate this issue and establish more reliable evidence.

In the subgroup analysis based on participants, the pooled HR of SO among community-dwelling people was 1.14 $(95 \% \mathrm{CI}=1.06-1.23)$, which was lower than the 1.65 HR (95\% CI $=1.17-2.33)$ of SO among hospitalized patients. This result may be explained by the fact that compared with hospitalized patients, community-dwelling individuals are more healthier and have well-preserved functional capacity. Another reason may be that hospitalized patients often have Coexistence state of activated inflammatory conditions and multiple comorbidities [42], which can give rise to higher levels of inflammatory factors, for instance, C-reactive proteins (CRP) and cytokines. Furthermore, according to a previous study, participants with sarcopenia have an increased level of serum inflammatory parameters,especially for CRP levels [43]. Therefore, these multiple risk factors form a vicious circle that increases the risk of death. Comprehensive diagnosis and treatment of SO should be performed much more attention for the hospitalized patients to reduce the progression of sarcopenia and improve their prognosis.

We performed a subgroup analysis based on obesity definition and found that participants with WC-based $\mathrm{SO}$ or BMI-based SO had a significantly increased risk of mortality compared with those without SO. However, the same result was not found for those with fat-massbased SO. It has been reported that fat mass cannot detect regional body fat, such as visceral fat, and that aging is related to an increase in visceral fat and a gradual decrease in muscle mass [44], which has an adverse effect on mortality [45]. This was found when obesity was measured by visceral fat: the pooled HR was 2.54 (95\% $\mathrm{CI}=1.83-3.53$ ), which was the highest HR in all the studies. Therefore, compared to other obesity definition variables for the elderly, visceral fat area is a good indicator of muscle-reduced obesity. 


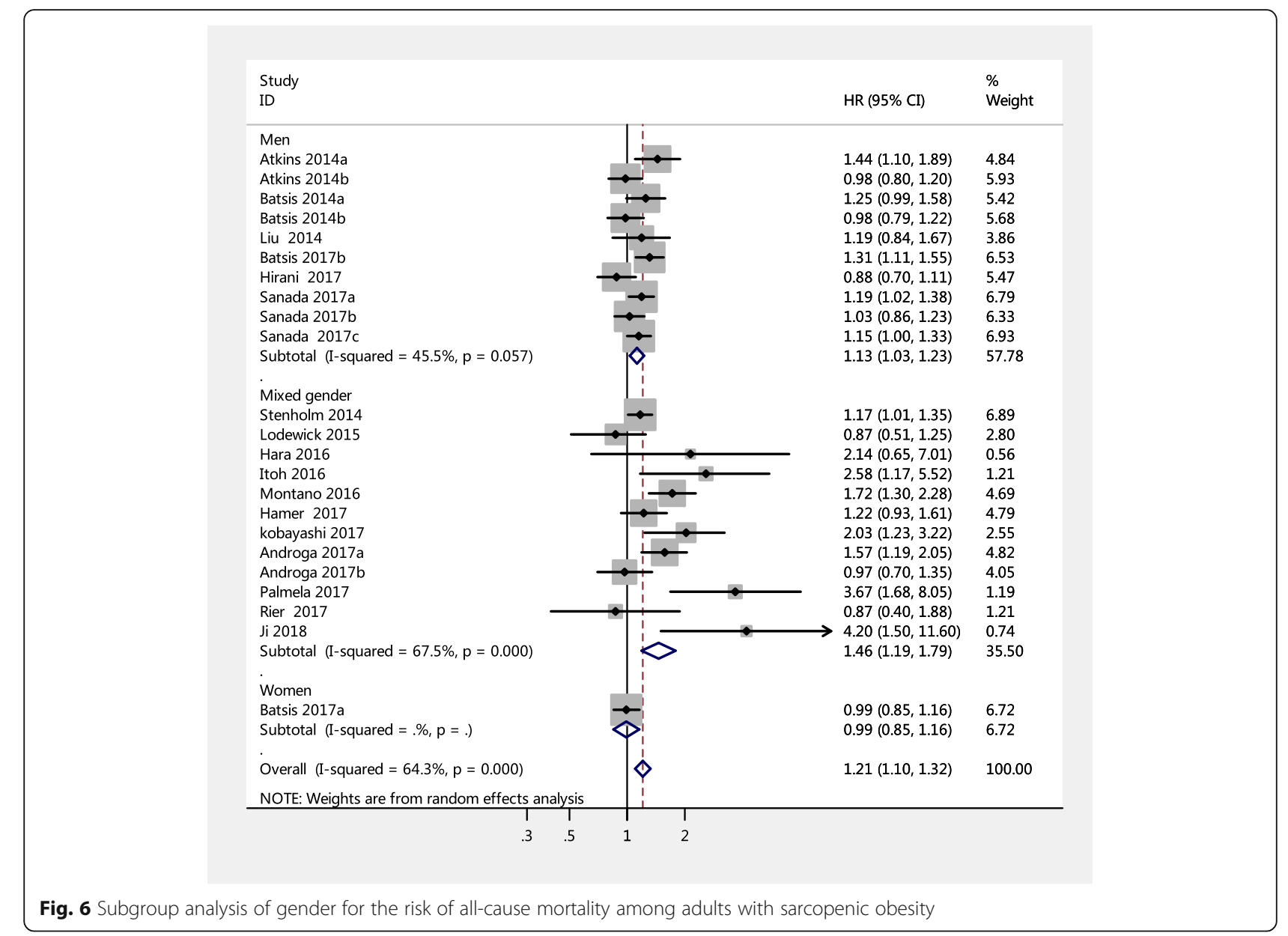

We were unable to draw a conclusion on the mechanisms underlying the relationship between $\mathrm{SO}$ and a higher risk of all-cause mortality. It was indicated that sarcopenia increases the risk of mortality among adults through symptoms such as low muscle mass [46], inflammation [47], insulin resistance, and myokines [11]. Above all the factors, low muscle mass might play the important role. Previous studies indicated low muscle mass may possible increasingly the risk of mortality [48, 49]. The factors mentioned above could affect survival through several mechanisms. In addition, preserving better muscles can help maintain major functional status and reduce the negative effects of falls, fractures, and sedentary lifestyles [50]. Stronger skeletal muscle mass can improve metabolism, enhance peripheral glucose treatment, and increase energy reserves, thereby decreasing the risk of mortality [51]. However, it is acknowledged that sarcoepnic adult was defined to have low muscle mass or poor physical performance, which is more likely to increase the risk of fall and fracture that aggravate the risk of mortality. Previous studies have shown that as obesity increases, skeletal muscle loss leads to an increase in inflammatory adipocytes, such as leptin, tumor necrosis factor alpha (TNF- $\alpha$ ), and interleukin (IL-6), and reduces concentrations of adiponectin or IL-15 [52]. In addition, the accumulation of visceral adipose tissue increases the amount of TNF- $\alpha$ and IL- 6 [53]. Moreover, excess visceral adipose tissue is seriously associated with increased insulin resistance [54]. Sarcopenia may influence important lifestyle habits, for instance poor dietary nutrient intake [55],declined physical activity [56], which cause $\mathrm{SO}$ in a vicious circle that deteriorate the situation of sarcopenia. All these changes may lead to adverse outcomes, especially mortality. In a word, $\mathrm{SO}$ is a geriatric syndrome rather than a disease; the mechanism of SO that leads to an increased risk of mortality is very complex and needs more research.

Our study has some limitations. First, a few of the included studies did not present the same confounding factors as those that were incorporated into the metaanalyses, which either underestimated or overestimated our results. For instance, physical activity is an important protective factor that alleviate the effect of $\mathrm{SO}$ on mortality, the HR of some including studies did not adjust physical activity. History of cancer or cardiovascular disease was another risk factor that could augment the 


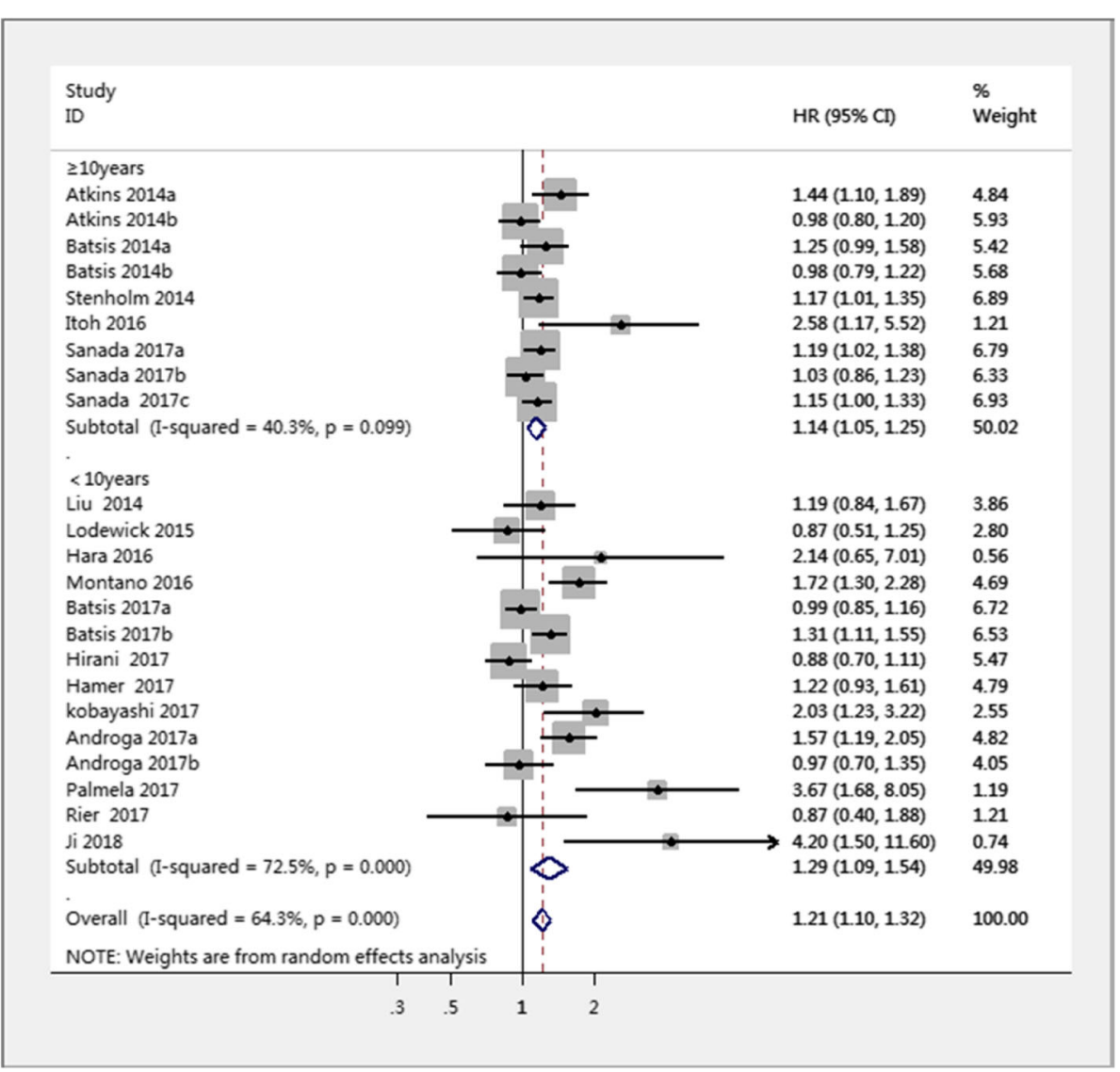

Fig. 7 Subgroup analysis of follow-up periods for the risk of all-cause among adults with sarcopenic obesity

negative impact of SO on mortality. Second, we only included research published in English, so data from important studies published in other languages may have been overlooked, which may lead to potential bias. However, these deficiencies do not reduce our contribution because the current study has multiple strengths. First, the original studies included in the study are all prospective designs that minimize the likelihood of recall bias and selection bias. Second, due to the large sample size, the current meta-analysis has reasonable statistical power, enabling us to explore the causal inference between SO and mortality. Third, this study carried out statistical analyses of sensitivity and publication bias that produced no statistically significant changes and no significant publication bias. Fourth, we conducted extensive subgroup analyses to make sure the results were more reliable.

\section{Conclusions}

In conclusion, based on systematic review and metaanalysis, it suggests that people with $\mathrm{SO}$ is an important predictor of all-caused mortality in adult people. The prevalence of SO is importantly increasing worldwide, therefore, it is very important to screen SO among people and nutrition and training exercise programs of prevention strategies are needed to preform, which could reduce the undesirable health outcomes associated with SO.

\section{Additional files}

Additional file 1: Search strategy of PubMed research report. (DOCX $11 \mathrm{~kb}$ ) Additional file 2: Figure S1. Funnel plot of publication bias of included studies. (DOCX $18 \mathrm{~kb}$ )

Additional file 3: Figure S2. Funnel plot of publication bias of included studies by trim-and-fill analyses. (DOCX $17 \mathrm{~kb}$ )

Additional file 4: Figure S3. Sensitivity analysis of all included studies. (DOCX $55 \mathrm{~kb}$ )

Additional file 5: Figure S4. Sensitivity analysis of age group. (DOC 34 kb)

\section{Abbreviations}

ALM/BMI: Appendicular lean mass/ body mass index; BF: Body fat; BMI: Body mass index; EWGSOP: European Working Group on Sarcopenia in Older People definition; FNIH: Foundation for the National Institutes of Health; MAMC: Midarm muscle circumference; MOOSE: Meta-analysis Of

Observational Studies in Epidemiology; MS: Muscle strength; SMI: Skeletal muscle index; SMM: Skeletal muscle mass; SO: Sarcopenic obesity; TNFa: Tumor necrosis factor alpha; WC: Waist circumference

\section{Acknowledgements}

The authors thank the staff of the Department of Emergency Medicine at the People's Hospital of Baoan ShenZhen for their guidance and support. 


\section{Authors' contributions}

$\mathrm{XMZ}$ and $\mathrm{XHX}$ were responsible for the data extraction and for producing the initial draft of the manuscript. QLD and RLD were responsible for the data extraction. WWZ was responsible for screening the papers and for quality assessment, and XMZ and CYL was responsible for screening the papers. YY was responsible for quality assessment and the statistical analysis. RLD and ASKC were responsible for the manuscript revision. All the authors approved the final version of the manuscript.

\section{Funding}

This work was supported by the Hospital Fund of the People's Hospital of Baoan ShenZhen (2018H006). The funders had no role in study design, data collection and analysis, decision to publish,and in writing the manuscript.

\section{Availability of data and materials}

All the data can be found in the electronic databases (PubMed, EMBASE, and the Cochrane Library).

\section{Ethics approval and consent to participate} Not applicable.

\section{Consent for publication}

Not applicable.

\section{Competing interests}

The authors declare that they have no competing interests.

\section{Author details}

'Department of Emergency, The Affiliated Baoan Hospital of Southern Medical University, The People's Hospital of Baoan Shenzhen, Shenzhen, China. ${ }^{2}$ Department of Nursing, The Affiliated Hospital of Shenzhen University, The Second People's Hospital of Shenzhen, Shenzhen, China. ${ }^{3}$ Department of Nursing, The Affiliated Baoan Hospital of Southern Medical University, People's Hospital of Baoan Shenzhen, Shenzhen, China. ${ }^{4}$ Department of Nursing, The Fifth Affiliated Hospital of Zunyi Medical University, Zhuhai, China. ${ }^{5}$ Department of Rehabilitation Sciences, The Hong Kong Polytechnic University, Hong Kong, China.

\section{Received: 16 November 2018 Accepted: 24 June 2019}

\section{Published online: 03 July 2019}

\section{References}

1. Tanimoto Y, Watanabe M, Sun W, Sugiura Y, Hayashida I, Kusabiraki T, Tamaki J. Sarcopenia and falls in community-dwelling elderly subjects in Japan: defining sarcopenia according to criteria of the European working group on sarcopenia in older people. Arch Gerontol Geriatr. 2014;59(2):295-9.

2. Steihaug OM, Gjesdal CG, Bogen B, Kristoffersen MH, Lien G, Ranhoff AH. Sarcopenia in patients with hip fracture: a multicenter cross-sectional study. PLoS One. 2017;12(9):e0184780.

3. Benjumea AM, Curcio CL, Duque G, Gomez F. Dynapenia and sarcopenia as a risk factor for disability in a falls and fractures clinic in older persons. Open Access Maced J Med Sci. 2018;6(2):344-9.

4. Chang KV, Hsu TH, Wu WT, Huang KC, Han DS. Is sarcopenia associated with depression? A systematic review and meta-analysis of observational studies. Age Ageing. 2017;46(5):738-46.

5. Giglio J, Kamimura MA, Lamarca F, Rodrigues J, Santin F, Avesani CM. Association of Sarcopenia with Nutritional Parameters, quality of life, hospitalization, and mortality rates of elderly patients on hemodialysis. J Ren Nutr. 2018:28(3):197-207.

6. Cawthon PM, Lui LY, Taylor BC, McCulloch CE, Cauley JA, Lapidus J, Orwoll E, Ensrud KE. Clinical definitions of sarcopenia and risk of hospitalization in community-dwelling older men: the osteoporotic fractures in men study. J Gerontol A Biol Sci Med Sci. 2017;72(10):1383-9.

7. Liu P, Hao Q, Hai S, Wang H, Cao L, Dong B. Sarcopenia as a predictor of allcause mortality among community-dwelling older people: a systematic review and meta-analysis. Maturitas. 2017;103:16-22.

8. Bluher M. Obesity: global epidemiology and pathogenesis. Nat Rev Endocrinol. 2019:15(5):288-98.

9. Dong SY, Wang ML, Li ZB, Dong Z, Liu YQ, Lu RJ, Li JM, Tang R. Obesity, weight change, and mortality in older adults with metabolic abnormalities. Nutr Metab Cardiovasc Dis. 2018;28(7):749-55.
10. Lv YB, Liu S, Yin ZX, Gao X, Kraus VB, Mao C, Yuan JQ, Zhang J, Luo JS, Chen $\mathrm{HS}$, et al. Associations of body mass index and waist circumference with 3year all-cause mortality among the oldest old: evidence from a Chinese community-based prospective cohort study. J Am Med Dir Assoc. 2018.

11. Kalinkovich A, Livshits G. Sarcopenic obesity or obese sarcopenia: a cross talk between age-associated adipose tissue and skeletal muscle inflammation as a main mechanism of the pathogenesis. Ageing Res Rev. 2017;35:200-21.

12. Zamboni M, Mazzali G, Fantin F, Rossi A, Di Francesco V. Sarcopenic obesity: a new category of obesity in the elderly. Nutr Metab Cardiovasc Dis. 2008; 18(5):388-95.

13. Kim JH, Lim S, Choi SH, Kim KM, Yoon JW, Kim KW, Lim JY, Park KS, Jang HC Sarcopenia: an independent predictor of mortality in community-dwelling older Korean men. J Gerontol A Biol Sci Med Sci. 2014;69(10):1244-52.

14. Whitlock G, Lewington S, Sherliker P, Clarke R, Emberson J, Halsey J, Qizilbash N, Collins R, Peto R. Body-mass index and cause-specific mortality in 900000 adults: collaborative analyses of 57 prospective studies. Lancet. 2009;373(9669):1083-96.

15. Sanada K, Chen R, Willcox B, Ohara T, Wen A, Takenaka C, Masaki K. Association of sarcopenic obesity predicted by anthropometric measurements and 24-y all-cause mortality in elderly men: the Kuakini Honolulu heart program. Nutrition. 2018;46:97-102.

16. Batsis JA, Mackenzie TA, Emeny RT, Lopez-Jimenez F, Bartels SJ. Low lean mass with and without obesity, and mortality: results from the 1999-2004 National Health and nutrition examination survey. J Gerontol A Biol Sci Med Sci. 2017;72(10):1445-51.

17. Liu L-K, Chen L-Y, Yeh K-P, Lin M-H, Hwang A-C, Peng L-N, Chen L-K. Sarcopenia, but not sarcopenic obesity, predicts mortality for older old men: a 3-year prospective cohort study. J Clin Gerontol Geriatr. 2014;5(2):42-6.

18. Hamer M, O'Donovan G. Sarcopenic obesity, weight loss, and mortality: the English longitudinal study of ageing. Am J Clin Nutr. 2017;106(1):125-9.

19. Tian S, Xu Y. Association of sarcopenic obesity with the risk of all-cause mortality: a meta-analysis of prospective cohort studies. Geriatr Gerontol Int. 2016;16(2):155-66

20. Van Aller C, Lara J, Stephan BCM, Donini LM, Heymsfield S, Katzmarzyk PT, Wells JCK, Prado CM, Siervo M. Sarcopenic obesity and overall mortality: results from the application of novel models of body composition phenotypes to the National Health and nutrition examination survey 19992004. Clin Nutr. 2018

21. Stroup DF, Berlin JA, Morton SC, Olkin I, Williamson GD, Rennie D, Moher D, Becker BJ, Sipe TA, Thacker SB. Meta-analysis of observational studies in epidemiology: a proposal for reporting. Meta-analysis of observational studies in epidemiology (MOOSE) group. Jama. 2000;283(15):2008-12.

22. Stenholm S, Mehta NK, Elo IT, Heliovaara M, Koskinen S, Aromaa A. Obesity and muscle strength as long-term determinants of all-cause mortality--a 33year follow-up of the Mini-Finland Health Examination Survey. Int J Obes. 2014;38(8):1126-32.

23. Hirani V, Naganathan V, Blyth F, Le Couteur DG, Seibel MJ, Waite LM, Handelsman DJ, Cumming RG. Longitudinal associations between body composition, sarcopenic obesity and outcomes of frailty, disability, institutionalisation and mortality in community-dwelling older men: the Concord health and ageing in men project. Age Ageing. 2017:46(3):413-20.

24. Atkins $\mathrm{J}$, Whincup $\mathrm{PH}$, Morris RW, Lennon LT, Papacosta O, Wannamethee SG. Sarcopenic obesity and risk of cardiovascular disease and mortality: a population-based cohort study of older men. J Am Geriatr Soc. 2014;62(2):253-60.

25. Batsis JA, Mackenzie TA, Barre LK, Lopez-Jimenez F, Bartels SJ. Sarcopenia, sarcopenic obesity and mortality in older adults: results from the National Health and nutrition examination survey III. Eur J Clin Nutr. 2014;68(9):1001-7.

26. Androga L, Sharma D, Amodu A, Abramowitz MK. Sarcopenia, obesity, and mortality in US adults with and without chronic kidney disease. Kidney Int Rep. 2017;2(2):201-11.

27. Hara N, Iwasa M, Sugimoto R, Mifuji-Moroka R, Yoshikawa K, Terasaka E, Hattori A, Ishidome M, Kobayashi Y, Hasegawa H, et al. Sarcopenia and Sarcopenic obesity are prognostic factors for overall survival in patients with cirrhosis. Intern Med. 2016;55(8):863-70

28. Itoh S, Yoshizumi T, Kimura K, Okabe H, Harimoto N, Ikegami T, Uchiyama H, Shirabe K, Nishie A, Maehara Y. Effect of Sarcopenic obesity on outcomes of living-donor liver transplantation for hepatocellular carcinoma. Anticancer Res. 2016;36(6):3029-34. 
29. Ji Y, Cheng B, Xu Z, Ye H, Lu W, Luo X, Fu S, Fang X. Impact of sarcopenic obesity on 30-day mortality in critically ill patients with intra-abdominal sepsis. J Crit Care. 2018;46:50-4.

30. Kobayashi A, Kaido T, Hamaguchi Y, et al. Impact of Sarcopenic Obesity on Outcomes in Patients Undergoing Hepatectomy for Hepatocellular Carcinoma. Ann Surg. 2019:269(5):924-31.

31. Lodewick TM, van Nijnatten TJ, van Dam RM, van Mierlo K, Dello SA Neumann UP, Olde Damink SW, Dejong CH. Are sarcopenia, obesity and sarcopenic obesity predictive of outcome in patients with colorectal liver metastases? HPB. 2015;17(5):438-46.

32. Montano-Loza AJ, Angulo P, Meza-Junco J, Prado CMM, Sawyer MB, Beaumont C, Esfandiari N, Ma M, Baracos VE. Sarcopenic obesity and myosteatosis are associated with higher mortality in patients with cirrhosis. J Cachexia Sarcopenia Muscle. 2016;7(2):126-35.

33. Palmela C, Velho S, Agostinho L, Branco F, Santos M, Santos MPC, Oliveira MH, Strecht J, Maio R, Cravo M, et al. Body composition as a prognostic factor of neoadjuvant chemotherapy toxicity and outcome in patients with locally advanced gastric cancer. J Gastric Cancer. 2017;17(1):74-87.

34. Rier HN, Jager A, Sleijfer S, van Rosmalen J, Kock M, Levin MD. Low muscle attenuation is a prognostic factor for survival in metastatic breast cancer patients treated with first line palliative chemotherapy. Breast. 2017;31:9-15.

35. Zhang X, Zhang W, Wang C, Tao W, Dou Q, Yang Y. Sarcopenia as a predictor of hospitalization among older people: a systematic review and meta-analysis. BMC Geriatr. 2018;18(1):188.

36. Prado CM, Lieffers JR, McCargar $L$, Reiman T, Sawyer MB, Martin L, Baracos VE. Prevalence and clinical implications of sarcopenic obesity in patients with solid tumours of the respiratory and gastrointestinal tracts: a population-based study. Lancet Oncol. 2008;9(7):629-35.

37. Landi F, Russo A, Liperoti R, Pahor M, Tosato M, Capoluongo E, Bernabei R, Onder G. Midarm muscle circumference, physical performance and mortality: results from the aging and longevity study in the Sirente geographic area (ilSIRENTE study). Clin Nutr. 2010;29(4):441-7.

38. Miller MD, Crotty M, Giles LC, Bannerman E, Whitehead C, Cobiac L, Daniels LA, Andrews G. Corrected arm muscle area: an independent predictor of long-term mortality in community-dwelling older adults? J Am Geriatr Soc. 2002;50(7):1272-7.

39. Muhlethaler R, Stuck AE, Minder CE, Frey BM. The prognostic significance of protein-energy malnutrition in geriatric patients. Age Ageing. 1995;24(3):193-7.

40. Dam TT, Peters KW, Fragala M, Cawthon PM, Harris TB, McLean R, Shardell M, Alley DE, Kenny A, Ferrucci L, et al. An evidence-based comparison of operational criteria for the presence of sarcopenia. J Gerontol A Biol Sci Med Sci. 2014;69(5):584-90.

41. Hars M, Biver E, Chevalley T, Herrmann F, Rizzoli R, Ferrari S, Trombetti A. Low lean mass predicts incident fractures independently from FRAX: a prospective cohort study of recent retirees. J Bone Miner Res. 2016; 31(11):2048-56.

42. Gariballa S, Alessa A. Sarcopenia: prevalence and prognostic significance in hospitalized patients. Clin Nutr. 2013;32(5):772-6.

43. Bano G, Trevisan C, Carraro S, Solmi M, Luchini C, Stubbs B, Manzato E, Serg G, Veronese N. Inflammation and sarcopenia: a systematic review and metaanalysis. Maturitas. 2017;96:10-5.

44. Yamada M, Moriguch Y, Mitani T, Aoyama T, Arai H. Age-dependent changes in skeletal muscle mass and visceral fat area in Japanese adults from 40 to 79 years-of-age. Geriatr Gerontol Int. 2014;14(Suppl 1):8-14

45. Pecorelli N, Carrara G, De Cobelli F, Cristel G, Damascelli A, Balzano G, Beretta L, Braga M. Effect of sarcopenia and visceral obesity on mortality and pancreatic fistula following pancreatic cancer surgery. Br J Surg. 2016; 103(4):434-42

46. Li R, Xia J, Zhang XI, Gathirua-Mwangi WG, Guo J, Li Y, McKenzie S, Song Y. Associations of muscle mass and strength with all-cause mortality among US older adults. Med Sci Sports Exerc. 2018;50(3):458-67.

47. Westbury LD, Fuggle NR, Syddall HE, Duggal NA, Shaw SC, Maslin K, Dennison EM, Lord JM, Cooper C. Relationships between markers of inflammation and muscle mass, strength and function: findings from the Hertfordshire cohort study. Calcif Tissue Int. 2018;102(3):287-95.

48. Brown JC, Harhay MO, Harhay MN. Appendicular lean mass and mortality among Prefrail and frail older adults. J Nutr Health Aging. 2017;21(3):342-5

49. Cesari M, Pahor M, Lauretani F, Zamboni V, Bandinelli S, Bernabei R, Guralnik $J M$, Ferrucci L. Skeletal muscle and mortality results from the InCHIANTI study. J Gerontol A Biol Sci Med Sci. 2009;64(3):377-84.
50. Cruz-Jentoft AJ, Bahat G, Bauer J, Boirie Y, Bruyere O, Cederholm T, Cooper C, Landi F, Rolland Y, Sayer AA, et al. Sarcopenia: revised European consensus on definition and diagnosis. Age Ageing. 2019;48(1):16-31.

51. Wolfe RR. The underappreciated role of muscle in health and disease. Am J Clin Nutr. 2006;84(3):475-82.

52. Lutz CT, Quinn LS. Sarcopenia, obesity, and natural killer cell immune senescence in aging: altered cytokine levels as a common mechanism. Aging (Albany NY). 2012;4(8):535-46.

53. Arano T, Nakagawa H, Tateishi R, Ikeda H, Uchino K, Enooku K, Goto E, Masuzaki R, Asaoka $Y$, Kondo $Y$, et al. Serum level of adiponectin and the risk of liver cancer development in chronic hepatitis $C$ patients. Int J Cancer. 2011;129(9):2226-35.

54. Srikanthan P, Hevener AL, Karlamangla AS. Sarcopenia exacerbates obesityassociated insulin resistance and dysglycemia: findings from the National Health and nutrition examination survey III. PLoS One. 2010;5(5):e10805.

55. Vandewoude MF, Alish CJ, Sauer AC, Hegazi RA. Malnutrition-sarcopenia syndrome: is this the future of nutrition screening and assessment for older adults? J Aging Res. 2012;2012:651570.

56. Yang CW, Li Cl, Li TC, Liu CS, Lin CH, Lin WY, Lin CC. The joint association of insulin sensitivity and physical activity on the skeletal muscle mass and performance in community-dwelling older adults. Exp Gerontol. 2017:95:34-8.

\section{Ready to submit your research? Choose BMC and benefit from:}

- fast, convenient online submission

- thorough peer review by experienced researchers in your field

- rapid publication on acceptance

- support for research data, including large and complex data types

- gold Open Access which fosters wider collaboration and increased citations

- maximum visibility for your research: over $100 \mathrm{M}$ website views per year

At $\mathrm{BMC}$, research is always in progress.

Learn more biomedcentral.com/submissions 